\title{
Mean square of the remainder term in the Dirichlet divisor problem II
}

\author{
by
}

\author{
KaI-Man Tsang (Hong Kong)
}

1. Introduction and main results. Let $d(n)$ denote the divisor function and let

$$
\Delta(x)=\sum_{n \leq x} d(n)-x(\log x+2 \gamma-1) \quad \text { for } x \geq 2
$$

be the error term in the Dirichlet divisor problem. The study of $\Delta(x)$ dates back at least to Dirichlet who first obtained by elementary argument the upper bound $\Delta(x) \ll x^{1 / 2}$ in 1838 . There has since been extensive work on the various properties of $\Delta(x)$ by many authors. See [4, Chapters 13, 14], [9, Chapter 12] and [6] for a comprehensive account of the subject.

In this paper we shall be concerned with an asymptotic formula for the mean square of $\Delta(x)$. In 1956, Tong [10] proved the classical result:

$$
\int_{2}^{X} \Delta(x)^{2} d x=\left(\left(6 \pi^{2}\right)^{-1} \sum_{m=1}^{\infty} d(m)^{2} m^{-3 / 2}\right) X^{3 / 2}+F(X)
$$

with $F(X) \ll X \log ^{5} X$. This shows that $\Delta(x)^{2}$ behaves nicely in the mean over intervals $[X, X+l]$ of length $l \gg \sqrt{X} \log ^{5} X$. Tong's result has resisted improvements for more than three decades until 1988, when Preissmann [8] obtained the slightly better bound

$$
F(X) \ll X \log ^{4} X,
$$

by employing a variant of Hilbert's inequality. (The same bound can also be obtained by using the estimate in Lemma 1 below. See [4, p. 98].) The improvement, though small, is not insignificant. It remains the best known upper bound for $F(X)$ in print and we shall see later why it is difficult to further reduce it.

The interest in the function $F(X)$ arises partly from its connection with the order of $\Delta(x)$. Ivić [4,Theorem3.8] observed that, if $U$ is an upper bound 
for $F(x)$ then $\Delta(x) \ll(U \log x)^{1 / 3}$ holds. Thus, from Hafner's $\Omega$-result [1]

$$
\Delta(x)=\Omega_{+}\left((x \log x)^{1 / 4}(\log \log x)^{(3+\log 4) / 4} e^{-A(\log \log \log x)^{1 / 2}}\right),
$$

where $A$ is a certain positive constant, Ivić deduced that

$$
F(x)=\Omega\left(x^{3 / 4}(\log x)^{-1 / 4}(\log \log x)^{3(3+\log 4) / 4} e^{-3 A(\log \log \log x)^{1 / 2}}\right) .
$$

(See also [5].) It was even optimistically conjectured that $F(x) \ll x^{3 / 4+\varepsilon}$ holds for any $\varepsilon>0$. This is a very strong conjecture since its truth would imply the long standing conjecture that $\Delta(x) \ll x^{1 / 4+\varepsilon}$ for any $\varepsilon>0$. In a previous paper [6] we disprove the above conjecture of Ivić by showing that

$$
F(x)=\Omega_{-}\left(x \log ^{2} x\right),
$$

which in turn is an immediate consequence of the formula [6, Theorem 2]:

$$
\int_{2}^{X} F(x) d x=-\left(8 \pi^{2}\right)^{-1} X^{2} \log ^{2} X+c X^{2} \log X+\mathcal{O}\left(X^{2}\right) .
$$

Here $c$ denotes a certain constant. This result closes up most of the gap between (1.1) and (1.2). It is the upper bound (1.1) rather than the lower bound (1.2) that is closer to the true order of magnitude of $F(x)$. This explains why further improvements on (1.1) are so difficult to obtain. The exact determination of the order of $F(x)$ seems to be very difficult and delicate.

The formula (1.3) can be reformulated as

$$
\int_{2}^{X}\left(F(x)+\left(4 \pi^{2}\right)^{-1} x \log ^{2} x-\kappa x \log x\right) d x \ll X^{2}
$$

for a certain constant $\kappa$. This suggests to us the

Conjecture.

$$
F(x)=-\left(4 \pi^{2}\right)^{-1} x \log ^{2} x+\kappa x \log x+\mathcal{O}(x) .
$$

As we have pointed out in [6], the $\mathcal{O}$-term here is best possible; it is oscillatory and cannot be $o(x)$. So, this conjecture offers the best possible asymptotic description for $F(x)$.

In this paper, we shall strengthen (1.4) and furnish strong evidence in support of our conjecture (1.5). We prove the following

MAIN THEOREM. There exist constants $\kappa$ and $c$ such that for any real number $r \geq 1$, we have

$$
\int_{2}^{X}\left|F(x)+\left(4 \pi^{2}\right)^{-1} x \log ^{2} x-\kappa x \log x\right|^{r} d x \ll(c r)^{4 r} X^{r+1} .
$$

The constant implied in the symbol $\ll$ is independent of $r$. 
Clearly (1.4) is a direct consequence of the case $r=1$. Furthermore, we have the following

COROLlARY. There is a positive constant $c^{\prime}$ such that for any increasing function $G(x)$ with $2 \leq G(x) \leq \log ^{4} x$, we have

$$
\left|F(x)+\left(4 \pi^{2}\right)^{-1} x \log ^{2} x-\kappa x \log x\right| \leq x G(x)
$$

for all but $\mathcal{O}\left(X \exp \left(-c^{\prime} G(X)^{1 / 4}\right)\right)$ values of $x$ in $[2, X]$. In particular, conjecture (1.5) holds true for almost all values of $x$, that is, the more precise asymptotic formula

$$
\begin{aligned}
\int_{2}^{X} \Delta(x)^{2} d x= & \left(\left(6 \pi^{2}\right)^{-1} \sum_{m=1}^{\infty} d(m)^{2} m^{-3 / 2}\right) X^{3 / 2} \\
& -\left(4 \pi^{2}\right)^{-1} X \log ^{2} X+\kappa X \log X+\mathcal{O}(X)
\end{aligned}
$$

is true for almost all $X \geq 2$.

The corollary follows easily from the Main Theorem by taking $r=$ $(e c)^{-1} G(X)^{1 / 4}$.

The plan of our proof of the Main Theorem is as follows: In Section 2 we shall prove four preliminary lemmas. Then we compute the expected main term for $F(x)$ in Section 3, and finally in Section 4, we bound the $r$ th power moments of four remainder terms resulting from Section 3.

2. Notations and some preparations. Throughout the paper, $\varepsilon$ denotes an arbitrary small positive number and $c$, a positive number, both of which may not be the same at each occurrence. The symbols $c_{0}, c_{1}, c_{2}, \ldots$ etc. denote certain unspecified constants. The well-known inequality: $d(n) \ll_{\varepsilon} n^{\varepsilon}$ for any $\varepsilon>0$ will be used freely without explicit reference. The constants implied in the symbols $\mathcal{O}$ and $\ll$ may depend on $\varepsilon$ only.

The method of proof of our Main Theorem builds on the idea in [6]. One of the main ingredients there is an asymptotic estimate for the sum

$$
\psi_{h}(y)=\sum_{m \leq y} d(m) d(m+h) \quad \text { for } y>0, h>0 .
$$

In connection with his work on the fourth power moment of the Riemann zeta-function on the critical line, Heath-Brown [2] proved the following.

LEMma 1. We have

$$
\psi_{h}(y)=I_{h}(y)+E_{h}(y)
$$

where the main term $I_{h}(y)$ is of the form

$$
I_{h}(y)=y \sum_{j=0}^{2} \log ^{j} y \sum_{d \mid h} d^{-1}\left(\alpha_{j 0}+\alpha_{j 1} \log d+\alpha_{j 2} \log ^{2} d\right)
$$


for certain constants $\alpha_{j i}$, and the remainder $E_{h}(y)$ satisfies

$$
E_{h}(y) \ll y^{5 / 6+\varepsilon}
$$

uniformly for $h \leq y^{5 / 6}$. In particular, $\alpha_{20}=6 \pi^{-2}, \alpha_{21}=\alpha_{22}=0$.

The bound (2.4) for $E_{h}(y)$ is not the sharpest known in the literature. For instance, Motohashi [7] has $E_{h}(y) \ll y^{2 / 3+\varepsilon}$ uniformly for $h \leq y^{20 / 27}$. But this does not help in reducing the bound in our Main Theorem and we are content with Heath-Brown's estimate. We note that $I_{h}(y)$ is roughly of the order $y \log ^{2} y$. In the computation of the main term for $F(x)$, we shall need $I_{h}^{\prime}(y)$, the derivative of $I_{h}(y)$. By $(2.3)$ we have

$$
I_{h}^{\prime}(y)=a_{2}(h) \log ^{2} y+a_{1}(h) \log y+a_{0}(h),
$$

where

$$
\begin{aligned}
& a_{2}(h)=6 \pi^{-2} \sum_{d \mid h} d^{-1}, \\
& a_{1}(h)=\sum_{d \mid h} d^{-1}\left(12 \pi^{-2}+\alpha_{10}+\alpha_{11} \log d+\alpha_{12} \log ^{2} d\right), \\
& a_{0}(h)=\sum_{d \mid h} d^{-1} \sum_{i=0}^{2}\left(\alpha_{0 i}+\alpha_{1 i}\right) \log ^{i} d .
\end{aligned}
$$

For any $y>0, Q>3$, let

$$
\xi(y, Q)=\sum_{h \leq y} h^{-1}\left(4 a_{2}(h) \log ^{2} h Q+2 a_{1}(h) \log h Q+a_{0}(h)\right) .
$$

We have proved in [6] the following.

Lemma 2. For any $y>0, Q>3$,

$$
\begin{aligned}
\xi(y, Q)= & \frac{4}{3} \log ^{3} y Q+c_{4} \log ^{2} y Q-\frac{4}{3} \log ^{3} Q+c_{5} \log ^{2} Q+c_{6} \log Q \\
& +c_{7} \log y+c_{8}+\mathcal{O}\left(y^{-1}\left|\log ^{3} y\right| \log ^{2} y Q\right) .
\end{aligned}
$$

Another salient feature in our argument is the use of the Bessel functions. For $\nu \geq 0$, let

$$
f(\nu)=\nu^{-1 / 2} J_{1 / 2}(\nu)-2 \nu^{-3 / 2} J_{3 / 2}(\nu),
$$

where $J_{k}$ denotes the Bessel function of order $k$. By the well-known estimate (for this and other properties of $J_{k}$, consult [11])

$$
J_{k}(z) \ll \min \left(|z|^{k},|z|^{-1 / 2}\right)
$$

for any real $z$, we have, for any $\nu \geq 0$,

$$
\begin{gathered}
f(\nu) \ll \min \left(1, \nu^{-1}\right), \\
f(\nu)=\nu^{-1 / 2} J_{1 / 2}(\nu)+\mathcal{O}\left(\nu^{-2}\right)=(2 / \pi)^{1 / 2} \nu^{-1} \sin \nu+\mathcal{O}\left(\nu^{-2}\right)
\end{gathered}
$$


and

$$
f^{\prime}(\nu)=-\nu^{-1 / 2} J_{3 / 2}(\nu)+2 \nu^{-3 / 2} J_{5 / 2}(\nu) \ll \nu^{-1} .
$$

Lemma 3. For $0 \leq a \leq 1 / 2,2 \leq A$ and $j=0,1,2,3$, we have

$$
\int_{a}^{A} f(\nu) \log ^{j} \nu d \nu=c_{j}+\mathcal{O}\left(a|\log a|^{j}+A^{-1} \log ^{j} A\right)
$$

with $c_{0}=0$ and $c_{1}=-2^{-3 / 2} \sqrt{\pi}$.

Proof. The argument in [6, Lemma 5] shows that

$$
\int_{0}^{\infty} f(\nu) \nu^{s} \log ^{j} \nu d \nu=\frac{d^{j}}{d s^{j}}\left\{-s 2^{s-3 / 2} \Gamma\left(\frac{1}{2}(s+1)\right) / \Gamma(2-s / 2)\right\}
$$

for $-1<\operatorname{Re} s<0$. Actually, this equation holds for all complex numbers $s$ which satisfy $|s|<1$. Indeed, for any $C>B>2$ we have, by (2.10),

$$
\begin{aligned}
\int_{B}^{C} f(\nu) & \nu^{s} \log ^{j} \nu d \nu \\
= & \left(\frac{2}{\pi}\right)^{1 / 2} \int_{B}^{C}(\sin \nu) \nu^{s-1} \log ^{j} \nu d \nu+\mathcal{O}\left(\int_{B}^{C} \nu^{-2}\left|\nu^{s}\right| \log ^{j} \nu d \nu\right) .
\end{aligned}
$$

Thus, uniformly for $|s|<1-\varepsilon$ we have

$$
\int_{B}^{C} f(\nu) \nu^{s} \log ^{j} \nu d \nu=\mathcal{O}_{\varepsilon}\left(B^{\operatorname{Re} s-1} \log ^{j} B\right),
$$

by applying partial integration to the first integral on the right hand side. This shows that the integral $\int_{0}^{\infty} f(\nu) \nu^{s} \log ^{j} \nu d \nu$ converges uniformly for $|s|<1-\varepsilon$ and hence defines an analytic function in the region $|s|<1$. Clearly, the right hand side of (2.12) is also analytic in the same region. So (2.12) holds for $|s|<1$. Equating both sides at $s=0$ then yields

$$
\int_{0}^{\infty} f(\nu) \log ^{j} \nu d \nu=c_{j}
$$

for $j=0,1,2,3$ with $c_{0}=0$ and $c_{1}=-2^{-3 / 2} \sqrt{\pi}$. Finally, by (2.13) at $s=0$, we have

$$
\int_{A}^{\infty} f(\nu) \log ^{j} \nu d \nu \ll A^{-1} \log ^{j} A
$$

and by (2.9),

$$
\int_{0}^{a} f(\nu) \log ^{j} \nu d \nu \ll \int_{0}^{a}\left|\log ^{j} \nu\right| d \nu \ll a|\log a|^{j}
$$


This proves our lemma.

Lemma 4. Let $y \geq 2$.

(a) If $0<\alpha<1$ and $\alpha+\beta \geq 2$ then

$$
\sum_{m \leq n \leq y} d(m) m^{-\alpha} d(n) n^{-\beta} \ll(1-\alpha)^{-1} \min \left(\log ^{3} y,(\alpha+\beta-2)^{-3}\right) .
$$

(b) If $\beta \geq 1$ then

$$
\sum_{h \leq m \leq y} d(m) m^{-\beta} d(m+h) h^{-1} \ll \min \left(\log ^{4} y,(\beta-1)^{-4}\right) .
$$

Proof. (a) For any $y \geq 2$,

$$
\begin{aligned}
\sum_{m \leq y} d(m) m^{-\alpha} & =\sum_{u \leq y} u^{-\alpha} \sum_{v \leq y / u} v^{-\alpha} \ll(1-\alpha)^{-1} \sum_{u \leq y} u^{-\alpha}(y / u)^{1-\alpha} \\
& \ll(1-\alpha)^{-1} y^{1-\alpha} \log y .
\end{aligned}
$$

Hence

$$
\begin{aligned}
\sum_{n \leq y}\left(\sum_{m \leq n} d(m) m^{-\alpha}\right) d(n) n^{-\beta} & \ll(1-\alpha)^{-1} \sum_{n \leq y} n^{1-\alpha-\beta} d(n) \log n \\
& =2(1-\alpha)^{-1} \sum_{u \leq y} u^{1-\alpha-\beta} \log u \sum_{v \leq y / u} v^{1-\alpha-\beta} .
\end{aligned}
$$

Since $1-\alpha-\beta \leq-1$, we have

$$
\sum_{u \leq y} u^{1-\alpha-\beta} \log u \sum_{v \leq y / u} v^{1-\alpha-\beta} \leq \sum_{u \leq y} u^{-1} \log u \sum_{v \leq y} v^{-1} \ll \log ^{3} y .
$$

On the other hand, for $1-\alpha-\beta<-1$,

$$
\begin{aligned}
\sum_{u \leq y} u^{1-\alpha-\beta} \log u \sum_{v \leq y / u} v^{1-\alpha-\beta} & \ll(\alpha+\beta-2)^{-1} \sum_{u \leq y} u^{1-\alpha-\beta} \log u \\
& \ll(\alpha+\beta-2)^{-3} .
\end{aligned}
$$

This proves part (a).

(b) We split the sum in part (b) into

$$
\begin{aligned}
\sum_{m \leq y} d(m) m^{-\beta} & \sum_{m^{5 / 6}<h \leq m} d(m+h) h^{-1} \\
& +\sum_{h \leq y^{5 / 6}} h^{-1} \sum_{h^{6 / 5}<m \leq y} d(m) d(m+h) m^{-\beta}=\Sigma_{1}+\Sigma_{2},
\end{aligned}
$$

say. For $\Sigma_{1}$ we use the asymptotic formula

$$
\sum_{m \leq t} d(m)=t(\log t+2 \gamma-1)+\mathcal{O}\left(t^{1 / 3}\right)
$$


and partial summation to show that

$$
\sum_{m^{5 / 6}<h \leq m} d(m+h) h^{-1} \ll \log ^{2} m .
$$

Then

$$
\begin{aligned}
\Sigma_{1} & \ll \sum_{m \leq y} d(m) m^{-\beta} \log ^{2} m=\sum_{u v \leq y}(u v)^{-\beta} \log ^{2}(u v) \\
& \leq 2 \sum_{u \leq v \leq y}(u v)^{-\beta} \log ^{2} v \ll \sum_{v \leq y} v^{-\beta} \log ^{3} v \\
& \ll \min \left(\log ^{4} y,(\beta-1)^{-4}\right) .
\end{aligned}
$$

For $\Sigma_{2}$ we use Lemma 1 and partial summation to obtain

$$
\sum_{h^{6 / 5}<m \leq y} d(m) d(m+h) m^{-\beta}=\int_{h^{6 / 5}}^{y} u^{-\beta} I_{h}^{\prime}(u) d u+\mathcal{O}\left(h^{-1 / 6}\right) .
$$

Then

$$
\Sigma_{2}=\int_{1}^{y} u^{-\beta} \sum_{h \leq u^{5 / 6}} h^{-1} I_{h}^{\prime}(u) d u+\mathcal{O}(1)
$$

By (2.5) we have

$$
\begin{aligned}
& \sum_{h \leq u^{5 / 6}} h^{-1} I_{h}^{\prime}(u) \\
= & \sum_{h \leq u^{5 / 6}} h^{-1} a_{2}(h) \log ^{2} u+\sum_{h \leq u^{5 / 6}} h^{-1} a_{1}(h) \log u+\sum_{h \leq u^{5 / 6}} h^{-1} a_{0}(h) .
\end{aligned}
$$

An elementary argument shows that

$$
\sum_{h \leq y} h^{-1} a_{j}(h) \ll \log y \quad \text { for } j=0,1,2
$$

(see $[6,(2.19)])$. Hence, by inserting this into $(2.16)$ we find from $(2.15)$ that

$$
\Sigma_{2} \ll \int_{1}^{y} u^{-\beta} \log ^{3} u d u+1 \ll \min \left(\log ^{4} y,(\beta-1)^{-4}\right) .
$$

This together with (2.14) proves part (b). Our lemma is thus established.

3. The main term of $F(x)$. Let $x \geq 2$ be any real number and $M=x^{7}$. Clearly, we may assume throughout that $x$ is large. Our starting point is the following formula $[6,(2.2)-(2.4)]$ :

$$
F(x)=S_{1}(x)+S_{2}(x)+\mathcal{O}(x),
$$


where

$$
\begin{aligned}
S_{1}(x)= & \left(2 \pi^{2}\right)^{-1} \sum_{m<n \leq M} d(m) d(n)(m n)^{-3 / 4} \\
& \times \int_{2}^{x} \sqrt{y} \cos (4 \pi(\sqrt{n}-\sqrt{m}) \sqrt{y}) d y
\end{aligned}
$$

and

$$
\begin{aligned}
S_{2}(x)= & \left(4 \pi^{2}\right)^{-1} \sum_{m, n \leq M} d(m) d(n)(m n)^{-3 / 4} \\
& \times \int_{2}^{x} \sqrt{y} \sin (4 \pi(\sqrt{n}+\sqrt{m}) \sqrt{y}) d y .
\end{aligned}
$$

We shall extract a main term from $S_{1}(x)$ and leave four remainder terms for Section 4.

By the well-known integral representation for the Bessel functions [11, Section 3.3]:

$$
J_{k+1 / 2}(z)=\frac{2}{\sqrt{\pi}}\left(\frac{z}{2}\right)^{k+1 / 2} \frac{1}{k !} \int_{0}^{1}\left(1-v^{2}\right)^{k} \cos (z v) d v, \quad k=0,1,2, \ldots,
$$

we find easily that, for any nonzero real number $\alpha$,

$$
\begin{aligned}
\int_{2}^{x} & \sqrt{y} \cos (\alpha \sqrt{y}) d y=2 x^{3 / 2} \int_{0}^{1} v^{2} \cos (\alpha \sqrt{x} v) d v-\int_{0}^{2} \sqrt{y} \cos (\alpha \sqrt{y}) d y \\
= & 2 x^{3 / 2}\left\{\sqrt{\pi}(2 \alpha \sqrt{x})^{-1 / 2} J_{1 / 2}(\alpha \sqrt{x})-\sqrt{2 \pi}(\alpha \sqrt{x})^{-3 / 2} J_{3 / 2}(\alpha \sqrt{x})\right\} \\
& -2 \int_{0}^{\sqrt{2}} u^{2} \cos \alpha u d u \\
= & \sqrt{2 \pi} x^{3 / 2} f(\alpha \sqrt{x})+\mathcal{O}\left(|\alpha|^{-1}\right),
\end{aligned}
$$

by (2.8). For convenience, write

$$
\theta_{m, n}=4 \pi \sqrt{x}(\sqrt{n}-\sqrt{m})
$$

and

$$
\phi_{m, n}=d(m) d(n)(m n)^{-3 / 4} f\left(\theta_{m, n}\right) .
$$

Then by (3.2) and (3.4),

$$
\begin{aligned}
S_{1}(x)= & \frac{1}{\sqrt{2}}\left(\frac{x}{\pi}\right)^{3 / 2} \sum_{m<n \leq M} \phi_{m, n} \\
& +\mathcal{O}\left(\sum_{m<n \leq M} d(m) d(n)(m n)^{-3 / 4}(\sqrt{n}-\sqrt{m})^{-1}\right) .
\end{aligned}
$$


The $\mathcal{O}$-term is

$$
\ll M^{\varepsilon} \sum_{m<n \leq M}(m n)^{-3 / 4} \sqrt{n}(n-m)^{-1} \ll M^{\varepsilon} \sum_{n \leq M} n^{-1} \log n \ll x^{\varepsilon},
$$

since $M=x^{7}$. Hence we can write

$$
S_{1}(x)=S_{3}(x)+S_{4}(x)+\mathcal{O}\left(x^{\varepsilon}\right)
$$

where

$$
S_{3}(x)=\frac{1}{\sqrt{2}}\left(\frac{x}{\pi}\right)^{3 / 2} \sum_{n / 2<m<n \leq M} \phi_{m, n}
$$

and

$$
S_{4}(x)=\frac{1}{\sqrt{2}}\left(\frac{x}{\pi}\right)^{3 / 2} \sum_{m \leq n / 2 \leq M / 2} \phi_{m, n} .
$$

In the double sum $S_{3}(x)$, we put $n=m+h$ with $h \geq 1$ and further decompose it into three sub-sums, namely,

$$
\begin{aligned}
S_{3}(x) & =\frac{1}{\sqrt{2}}\left(\frac{x}{\pi}\right)^{3 / 2} \sum_{h \leq M / 2} \sum_{h<m \leq M-h} \phi_{m, m+h} \\
& =S_{5}(x)+S_{6}(x)+S_{7}(x),
\end{aligned}
$$

where

$$
\begin{aligned}
S_{5}(x) & =\frac{1}{\sqrt{2}}\left(\frac{x}{\pi}\right)^{3 / 2} \sum_{h \leq H} \sum_{D<m \leq M-h} \phi_{m, m+h}, \\
S_{6}(x) & =\frac{1}{\sqrt{2}}\left(\frac{x}{\pi}\right)^{3 / 2} \sum_{h \leq H} \sum_{h<m \leq D} \phi_{m, m+h}, \\
S_{7}(x) & =\frac{1}{\sqrt{2}}\left(\frac{x}{\pi}\right)^{3 / 2} \sum_{H<h \leq M / 2} \sum_{h<m \leq M-h} \phi_{m, m+h}, \\
H & =x^{\tau}, \quad \tau=49 / 16,
\end{aligned}
$$

and for each $h \leq H$,

$$
D=D_{h}=(h \sqrt{x})^{7 / 4} .
$$

We now proceed to evaluate the sum $S_{5}(x)$ which contains the main term of $F(x)$. 
By Riemann-Stieltjes integration, (2.1), (2.2) and (3.6), we have

$$
\begin{aligned}
\sum_{D<m \leq M-h} \phi_{m, m+h}= & \int_{D}^{M-h}(y(y+h))^{-3 / 4} f\left(\theta_{y, y+h}\right) d \psi_{h}(y) \\
= & \int_{D}^{M-h}(y(y+h))^{-3 / 4} f\left(\theta_{y, y+h}\right) I_{h}^{\prime}(y) d y \\
& +\left.E_{h}(y)(y(y+h))^{-3 / 4} f\left(\theta_{y, y+h}\right)\right|_{D} ^{M-h} \\
& -\int_{D}^{M-h} E_{h}(y) \frac{d}{d y}\left\{(y(y+h))^{-3 / 4} f\left(\theta_{y, y+h}\right)\right\} d y \\
= & W_{1}(h)+W_{2}(h)-W_{3}(h),
\end{aligned}
$$

say. Note that

$$
\theta_{y, y+h}=4 \pi \sqrt{x}(\sqrt{y+h}-\sqrt{y}) \approx 2 \pi h \sqrt{x / y} .
$$

Hence by (2.9),

$$
f\left(\theta_{y, y+h}\right) \ll \sqrt{y} h^{-1} x^{-1 / 2} .
$$

As $D>h^{6 / 5}$, we may apply $(2.4)$ to bound $E_{h}(y)$ in $W_{2}(h)$ and $W_{3}(h)$. So by (3.15) and (3.14), we have

$$
W_{2}(h) \ll D^{-1 / 6+\varepsilon} h^{-1} x^{-1 / 2} \ll(h \sqrt{x})^{-5 / 4} .
$$

With the help of (2.11) and (2.9), similar argument yields

$$
\begin{aligned}
W_{3}(h) & \ll \int_{D}^{M-h} y^{5 / 6+\varepsilon}\left\{y^{-5 / 2}+y^{-3 / 2} \sqrt{y} h^{-1} x^{-1 / 2} h \sqrt{x} y^{-3 / 2}\right\} d y \\
& \ll D^{-2 / 3+\varepsilon} \ll(h \sqrt{x})^{-13 / 12} .
\end{aligned}
$$

Whence

$$
\sum_{D<m \leq M-h} \phi_{m, m+h}=W_{1}(h)+\mathcal{O}\left((h \sqrt{x})^{-13 / 12}\right) .
$$

To evaluate the integral

$$
W_{1}(h)=\int_{D}^{M-h}(y(y+h))^{-3 / 4} f\left(\theta_{y, y+h}\right) I_{h}^{\prime}(y) d y,
$$

we make the change of variable:

$$
\omega=\theta_{y, y+h}=4 \pi \sqrt{x}(\sqrt{y+h}-\sqrt{y}) .
$$

Then $\omega$ lies in the interval $\left[u_{1}, u_{2}\right]$ where

$$
u_{1}=4 \pi \sqrt{x}(\sqrt{M}-\sqrt{M-h})=2 \pi h x^{-3}+\mathcal{O}\left(h^{2} x^{-10}\right)
$$


and

$$
u_{2}=4 \pi \sqrt{x}(\sqrt{D+h}-\sqrt{D}) \approx 2 \pi h \sqrt{x} D^{-1 / 2} \geq 2 \pi H x^{-3},
$$

by (3.14). Moreover, we notice that $\omega^{2} x^{-1} h^{-1} \ll x^{-7 / 8}$ for $\omega \in\left[u_{1}, u_{2}\right]$.

Thus by (3.17) we find that

$$
y=4 \pi^{2} x \omega^{-2} h^{2}-\frac{1}{2} h+\left(64 \pi^{2} x\right)^{-1} \omega^{2}=4 \pi^{2} x h^{2} \omega^{-2}\left(1+\mathcal{O}\left(\omega^{2} x^{-1} h^{-1}\right)\right),
$$

so that

$$
\begin{aligned}
(y(y+h))^{-3 / 4} & =\left(4 \pi^{2} x h^{2} \omega^{-2}-\left(64 \pi^{2} x\right)^{-1} \omega^{2}\right)^{-3 / 2} \\
& =\omega^{3}(2 \pi h \sqrt{x})^{-3}\left(1+\mathcal{O}\left(\omega^{4} x^{-2} h^{-2}\right)\right)
\end{aligned}
$$

and

$$
\frac{d y}{d \omega}=-8 \pi^{2} x h^{2} \omega^{-3}\left(1+\mathcal{O}\left(\omega^{4} x^{-2} h^{-2}\right)\right)
$$

Moreover, by (2.5) and (2.6) (which shows $a_{0}(h), a_{1}(h) \ll \log ^{3} h$ and $a_{2}(h)$ $\ll \log h)$ we have

$$
I_{h}^{\prime}(y)=B_{h}(\omega)+\mathcal{O}\left(\omega^{2} x^{-1} h^{-1} \log ^{3} x\right),
$$

where

(3.20) $B_{h}(\omega)=4 a_{2}(h) \log ^{2}\left(2 \pi h \sqrt{x} \omega^{-1}\right)+2 a_{1}(h) \log \left(2 \pi h \sqrt{x} \omega^{-1}\right)+a_{0}(h)$.

With all these approximations we find that

$$
W_{1}(h)=(\pi \sqrt{x})^{-1} \int_{u_{1}}^{u_{2}} f(\omega) h^{-1}\left(B_{h}(\omega)+\mathcal{O}\left(\omega^{2} x^{-1} h^{-1} \log ^{3} x\right)\right) d \omega .
$$

By (2.9), (3.19) and (3.14) the $\mathcal{O}$-term in the integrand contributes no more than

$$
h^{-2} x^{-3 / 2} \log ^{3} x \int_{u_{1}}^{u_{2}} \min \left(1, \omega^{-1}\right) \omega^{2} d \omega \ll x^{-1 / 2} D^{-1} \log ^{3} x \leq x^{-1 / 2} h^{-7 / 4} .
$$

Moreover, by (3.18), when we replace the lower integration limit by $2 \pi h x^{-3}$, the error induced in $W_{1}(h)$ is

$$
\ll(h \sqrt{x})^{-1} h^{2} x^{-10}\left(a_{2}(h) \log ^{2} x+\left|a_{1}(h)\right| \log x+\left|a_{0}(h)\right|\right) \ll h x^{-21 / 2} \log ^{4} x .
$$

Similarly, by (2.10), if we change the upper integration limit to $2 \pi H x^{-3}$, the error induced in $W_{1}(h)$ is bounded by

$$
(h \sqrt{x})^{-1}\left|\int_{2 \pi H x^{-3}}^{u_{2}}\left(\omega^{-1} \sin \omega+\mathcal{O}\left(\omega^{-2}\right)\right) B_{h}(\omega) d \omega\right| .
$$

The contribution of the term $\mathcal{O}\left(\omega^{-2}\right)$ is $\ll(h \sqrt{x})^{-1}\left(H x^{-3}\right)^{-1} \log ^{4} x$, while 
by partial integration

$$
\begin{aligned}
\int_{2 \pi H x^{-3}}^{u_{2}} \omega^{-1} \sin \omega B_{h}(\omega) d \omega \\
\quad=\mathcal{O}\left(\left(H x^{-3}\right)^{-1} \log ^{4} x\right)+\int_{2 \pi H x^{-3}}^{u_{2}} \cos \omega \frac{d}{d \omega}\left\{\omega^{-1} B_{h}(\omega)\right\} d \omega \\
= \\
=\mathcal{O}\left(\left(H x^{-3}\right)^{-1} \log ^{4} x\right)+\mathcal{O}\left(\int_{2 \pi H x^{-3}}^{u_{2}} \omega^{-2} \log ^{4} x d \omega\right) \\
=\mathcal{O}\left(\left(H x^{-3}\right)^{-1} \log ^{4} x\right) .
\end{aligned}
$$

Hence the expression in (3.21) is

$$
\ll(h \sqrt{x})^{-1}\left(H x^{-3}\right)^{-1} \log ^{4} x \ll x^{-9 / 16} h^{-1} \log ^{4} x,
$$

by (3.13). With all these estimations and simplifications, we can now write

$$
\begin{aligned}
W_{1}(h)= & (\pi \sqrt{x})^{-1} \int_{2 \pi h x^{-3}}^{2 \pi H x^{-3}} f(\omega) h^{-1} B_{h}(\omega) d \omega \\
& +\mathcal{O}\left(x^{-1 / 2} h^{-7 / 4}+x^{-21 / 2} h \log ^{4} x+x^{-9 / 16} h^{-1} \log ^{4} x\right) .
\end{aligned}
$$

Then by (3.16), (3.10) and (3.13), we have

$$
S_{5}(x)=\left(2 \pi^{5}\right)^{-1 / 2} x \sum_{h \leq H} \int_{2 \pi h x^{-3}}^{2 \pi H x^{-3}} f(\omega) h^{-1} B_{h}(\omega) d \omega+\mathcal{O}(x) .
$$

Interchanging the summation and integration, and in view of (2.7), (3.20) we find that

$$
S_{5}(x)=\left(2 \pi^{5}\right)^{-1 / 2} x \int_{2 \pi x^{-3}}^{2 \pi H x^{-3}} f(\omega) \xi\left(\frac{x^{3} \omega}{2 \pi}, \frac{2 \pi \sqrt{x}}{\omega}\right) d \omega+\mathcal{O}(x) .
$$

Now by Lemma 2 ,

$$
\begin{aligned}
\xi\left(\frac{x^{3} \omega}{2 \pi}, \frac{2 \pi \sqrt{x}}{\omega}\right)= & \log \omega \log ^{2} x+\left(c_{9} \log \omega+c_{10} \log ^{2} \omega\right) \log x \\
& +\Phi_{1}(\log \omega)+\Phi_{2}(\log x)+\mathcal{O}\left(\omega^{-1} x^{-3} \log ^{5} x\right),
\end{aligned}
$$

where $\Phi_{1}, \Phi_{2}$ are certain polynomials of degrees at most 3 . Thus by Lemma 3 ,

$$
\begin{aligned}
\int_{2 \pi x^{-3}}^{2 \pi H x^{-3}} f(\omega) \xi\left(\frac{x^{3} \omega}{2 \pi}, \frac{2 \pi \sqrt{x}}{\omega}\right) d \omega= & -2^{-3 / 2} \sqrt{\pi} \log ^{2} x+c_{11} \log x+c_{12} \\
& +\mathcal{O}\left(\left(x^{3} H^{-1}+x^{-3}\right) \log ^{3} x+x^{-3} \log ^{6} x\right) .
\end{aligned}
$$


Inserting this into (3.22) and in view of (3.13), we have

$$
S_{5}(x)=-\left(4 \pi^{2}\right)^{-1} x \log ^{2} x+\kappa x \log x+\mathcal{O}(x)
$$

for a certain constant $\kappa$. Combining this with (3.1), (3.7) and (3.9), we have proved that

$$
\begin{aligned}
F(x)+\left(4 \pi^{2}\right)^{-1} x \log ^{2} x-\kappa x \log x \\
\quad=S_{2}(x)+S_{4}(x)+S_{6}(x)+S_{7}(x)+\mathcal{O}(x) .
\end{aligned}
$$

4. Completion of the proof of Main Theorem. We are now ready to complete the proof of our Main Theorem.

Let $X$ be any large number (which is independent of $r$ ). In view of (3.23), it is sufficient to prove

$$
\int_{2}^{X}\left|S_{j}(x)\right|^{r} d x \ll(c r)^{4 r} X^{r+1} \quad \text { for } j=2,4,6,7,
$$

in order to obtain our Main Theorem. Recall that the symbol $c$ denotes a certain positive constant which may not be the same at each occurrence.

Let $\delta=10^{-4}$. It is easy to verify that

$$
X^{-\delta} \log ^{4 r} X \leq\left(\frac{4 r}{e \delta}\right)^{4 r} .
$$

Hence in the course of our analysis, errors and bounds of the form $X^{1+r-\delta}(c \log X)^{4 r}$ are acceptable. Furthermore, by Hölder's inequality, we need only to prove $(4.1)_{j}$ for positive even integers $r$.

Consider first the easiest case $(4.1)_{2}$, that is, the bound

$$
\int_{2}^{X}\left(S_{2}(x)\right)^{r} d x \ll(c r)^{4 r} X^{r+1} .
$$

Recall from (3.3) the definition of $S_{2}(x)$. Since

$$
\int \sqrt{y} \sin (\alpha \sqrt{y}) d y=-2 y \alpha^{-1} \cos \alpha \sqrt{y}+4 \sqrt{y} \alpha^{-2} \sin \alpha \sqrt{y}+4 \alpha^{-3} \cos \alpha \sqrt{y}
$$

for any $\alpha \neq 0$, we can rewrite

$$
\begin{gathered}
S_{2}(x)=-(2 \pi)^{-3} x \sum_{m, n \leq M} d(m) d(n)(m n)^{-3 / 4}(\sqrt{n}+\sqrt{m})^{-1} \\
\quad \times \cos (4 \pi(\sqrt{n}+\sqrt{m}) \sqrt{x}) \\
+\mathcal{O}\left(\sum_{m, n \leq M} d(m) d(n)(m n)^{-3 / 4}\left(\sqrt{x}(\sqrt{n}+\sqrt{m})^{-2}+(\sqrt{n}+\sqrt{m})^{-1}\right)\right) .
\end{gathered}
$$

The $\mathcal{O}$-term here is

$$
\ll \sqrt{x} \sum_{m \leq n \leq M} d(m) d(n) m^{-3 / 4} n^{-7 / 4}+\sum_{m \leq n \leq M} d(m) d(n) m^{-3 / 4} n^{-5 / 4} \ll \sqrt{x},
$$


by Lemma 4(a). Hence

$$
\begin{array}{rl}
\int_{2}^{X}\left(S_{2}(x)\right)^{r} & d x \ll c^{r} \sum_{m_{i}, n_{j} \leq X^{7}} \prod_{i=1}^{r} b\left(m_{i}, n_{i}\right) \\
& \times \int_{\max _{i, j}\left(m_{i}, n_{j}\right)^{1 / 7}}^{X} x^{r} \prod_{i=1}^{r} \cos \left(4 \pi \sigma_{i} \sqrt{x}\right) d x+\mathcal{O}\left((c X)^{r / 2+1}\right),
\end{array}
$$

where $\sigma_{i}=\sqrt{n_{i}}+\sqrt{m_{i}}$ and

$$
b(m, n)=d(m) d(n)(m n)^{-3 / 4}(\sqrt{n}+\sqrt{m})^{-1} .
$$

The product $\prod_{i=1}^{r} \cos \left(4 \pi \sigma_{i} \sqrt{x}\right)$ is equal to a sum of $2^{r-1}$ terms, each of which is of the form $2^{-r+1} \cos \left(4 \pi \sqrt{x}\left(\sigma_{1} \pm \sigma_{2} \pm \ldots \pm \sigma_{r}\right)\right)$. By the second mean value theorem (see [9, Lemma 4.3]),

$$
\int_{X_{0}}^{X} x^{r} e^{i \alpha \sqrt{x}} d x \ll \min \left(X^{r+1}, X^{r+1 / 2}|\alpha|^{-1}\right)
$$

for any $X_{0}>0$ and any real number $\alpha$. Applying this we find that

$$
\begin{aligned}
\int_{\max _{i, j}\left(m_{i}, n_{j}\right)^{1 / 7}}^{X} & x^{r} \prod_{i=1}^{r} \cos \left(4 \pi \sigma_{i} \sqrt{x}\right) d x \\
& \ll 2^{-r} \sum_{ \pm} \min \left(X^{r+1}, X^{r+1 / 2}\left|\sigma_{1} \pm \sigma_{2} \pm \ldots \pm \sigma_{r}\right|^{-1}\right),
\end{aligned}
$$

where the last summation $\sum_{ \pm}$is over all the $2^{r-1}$ possible combinations of the \pm signs. In view of (4.5), the bound (4.3) would follow once we establish the inequality

$$
\begin{array}{r}
\sum_{m_{i}, n_{j} \leq X^{7}} \prod_{i=1}^{r} b\left(m_{i}, n_{i}\right) \min \left(X^{r+1}, X^{r+1 / 2}\left|\sigma_{1} \pm \sigma_{2} \pm \ldots \pm \sigma_{r}\right|^{-1}\right) \\
\ll(c r)^{4 r} X^{r+1}
\end{array}
$$

for each combination of the \pm signs. Let us now consider the left hand side of (4.8) for a fixed combination of the \pm signs. We may, in addition, assume $n_{1}$ to be the largest among all the $n_{i}, m_{j}$ in the summation. Write $\sigma^{\prime}= \pm \sigma_{2} \pm \ldots \pm \sigma_{r}$ for short. Then on considering the two cases: $\left|\sigma_{1}+\sigma^{\prime}\right|>1$ and $\left|\sigma_{1}+\sigma^{\prime}\right| \leq 1$, we see that the sum on the left hand side of (4.8) is less than or equal to

$$
\begin{array}{r}
X^{r+1 / 2} \sum_{m_{i}, n_{j} \leq X^{7}} \prod_{i=1}^{r} b\left(m_{i}, n_{i}\right)+X^{r+1} \sum_{\substack{m_{i}, n_{j} \leq X^{7} \\
\left|\sigma_{1}+\sigma^{\prime}\right| \leq 1}} \prod_{i=1}^{r} b\left(m_{i}, n_{i}\right) \\
=X^{r+1 / 2} \Sigma^{\prime}+X^{r+1} \Sigma^{\prime \prime},
\end{array}
$$


say. By Lemma 4(a) and (4.6),

$$
\begin{aligned}
\Sigma^{\prime} & =\left(\sum_{m, n \leq X^{7}} b(m, n)\right)^{r} \\
& \ll c^{r}\left(\sum_{m \leq n \leq X^{7}} d(m) m^{-3 / 4} d(n) n^{-5 / 4}\right)^{r} \ll\left(c \log ^{3} X\right)^{r} .
\end{aligned}
$$

In $\Sigma^{\prime \prime}$, for given $m_{2}, \ldots, m_{r}, n_{1}, \ldots, n_{r}$ the condition $\left|\sigma_{1}+\sigma^{\prime}\right| \leq 1$ stipulates that $\sqrt{m_{1}}$ must lie in an interval $[v, v+2]$ for some $v=v\left(m_{2}, \ldots, n_{r}\right)$ with $v \ll \sqrt{n_{1}}$. Hence $m_{1}$ is determined up to $\mathcal{O}\left(\sqrt{n_{1}}\right)$ consecutive values so that

$$
\sum_{m_{1}} d\left(m_{1}\right) m_{1}^{-3 / 4} \leq \sum_{m_{1} \ll \sqrt{n_{1}}} m_{1}^{-3 / 4+\delta} \ll n_{1}^{1 / 8+\delta} .
$$

Then

$$
\begin{aligned}
\Sigma^{\prime \prime} & \leq \sum_{\substack{n_{i}, m_{i} \leq X^{7} \\
i \geq 2}} \prod_{i=2}^{r} b\left(m_{i}, n_{i}\right) \sum_{n_{1}} d\left(n_{1}\right) n_{1}^{-5 / 4} n_{1}^{1 / 8+\delta} \\
& \ll \sum_{\substack{n_{i}, m_{i} \leq X^{7} \\
i \geq 2}} \prod_{i=2}^{r} b\left(m_{i}, n_{i}\right)\left(\max _{i \geq 2}\left(n_{i}, m_{i}\right)\right)^{-1 / 9},
\end{aligned}
$$

since $n_{1} \geq \max _{i \geq 2}\left(n_{i}, m_{i}\right)$. Consequently,

$$
\begin{aligned}
\Sigma^{\prime \prime} & \ll \sum_{n_{i}, m_{i} \leq X^{7}} \prod_{i=2}^{r}\left(b\left(m_{i}, n_{i}\right) \max \left(n_{i}, m_{i}\right)^{-1 /(9 r)}\right) \\
& \ll c^{r}\left(\sum_{m \leq n \leq X^{7}} d(m) m^{-3 / 4} d(n) n^{-5 / 4-1 /(9 r)}\right)^{r-1} \ll c^{r} r^{3 r},
\end{aligned}
$$

by (4.6) and Lemma 4(a). Combining this estimate with that in (4.10), we find that the right hand side of (4.9) is bounded by $X^{r+1 / 2}\left(c \log ^{3} X\right)^{r}+$ $X^{r+1}(c r)^{3 r}$ which, in view of (4.2), yields the inequality (4.8).

Next we consider $(4.1)_{4}$, that is, the inequality

$$
\int_{2}^{X}\left(S_{4}(x)\right)^{r} d x \ll(c r)^{4 r} X^{r+1} .
$$

By (3.8), (3.6) and (2.10), we can write

$$
\begin{aligned}
S_{4}(x)= & \pi^{-2} x^{3 / 2} \sum_{m \leq n / 2 \leq M / 2} d(m) d(n)(m n)^{-3 / 4} \theta_{m, n}^{-1} \sin \theta_{m, n} \\
& +\mathcal{O}\left(x^{3 / 2} \sum_{m \leq n / 2 \leq M / 2} d(m) d(n)(m n)^{-3 / 4} \theta_{m, n}^{-2}\right) .
\end{aligned}
$$


By (3.5) and Lemma 4(a), the $\mathcal{O}$-term is

$$
\begin{aligned}
& \ll \sqrt{x} \sum_{m \leq n / 2 \leq M / 2} d(m) d(n)(m n)^{-3 / 4}(\sqrt{n}-\sqrt{m})^{-2} \\
& \ll \sqrt{x} \sum_{m \leq n / 2 \leq M / 2} d(m) m^{-3 / 4} d(n) n^{-7 / 4} \ll \sqrt{x} .
\end{aligned}
$$

Hence

$$
\begin{aligned}
S_{4}(x)= & \left(4 \pi^{3}\right)^{-1} x \sum_{m \leq n / 2 \leq M / 2} d(m) d(n)(m n)^{-3 / 4}(\sqrt{n}-\sqrt{m})^{-1} \\
& \times \sin (4 \pi \sqrt{x}(\sqrt{n}-\sqrt{m}))+\mathcal{O}(\sqrt{x}) .
\end{aligned}
$$

The double sum here can be compared with that for $S_{2}(x)$ in (4.4). Here we have the factor $\sqrt{n}-\sqrt{m}$ instead of $\sqrt{n}+\sqrt{m}$. But since $m \leq n / 2$, $\sqrt{n}-\sqrt{m}$ has the same order as $\sqrt{n}+\sqrt{m}$. Thus, the foregoing analysis can be carried over and we obtain in much the same way the bound (4.11).

The proof of the remaining two inequalities in (4.1) requires a bit more effort. We consider first the bound $(4.1)_{6}$, that is,

$$
\int_{2}^{X}\left(S_{6}(x)\right)^{r} d x \ll(c r)^{4 r} X^{r+1} .
$$

Recall from (3.11) and (3.6) the definition of $S_{6}(x)$. Firstly, similar to the treatment of $S_{4}(x)$, we make the substitution

$$
f\left(\theta_{m, m+h}\right)=(2 / \pi)^{1 / 2} \theta_{m, m+h}^{-1} \sin \theta_{m, m+h}+\mathcal{O}\left(\theta_{m, m+h}^{-2}\right)
$$

in $S_{6}(x)$. Then

$$
\begin{aligned}
S_{6}(x)= & \pi^{-2} x^{3 / 2} \sum_{h \leq H} \sum_{h<m \leq D} d(m) d(m+h)(m(m+h))^{-3 / 4} \\
& \times \theta_{m, m+h}^{-1} \sin \theta_{m, m+h} \\
+ & \mathcal{O}\left(\sqrt{x} \sum_{h \leq H} \sum_{h<m \leq D} d(m) d(m+h)(m(m+h))^{-3 / 4}(\sqrt{m+h}-\sqrt{m})^{-2}\right) .
\end{aligned}
$$

Since $\sqrt{m+h}-\sqrt{m} \approx \frac{1}{2} h m^{-1 / 2}$, the $\mathcal{O}$-term is

$$
\ll \sqrt{x} \sum_{h \leq H} h^{-2} \sum_{h<m \leq D} d(m) d(m+h) m^{-1 / 2} \ll \sqrt{x} \sum_{h \leq H} h^{-2} D^{1 / 2+\varepsilon} \ll x,
$$

by (3.14). Hence 


$$
\begin{aligned}
\int_{2}^{X}\left(S_{6}(x)\right)^{r} d x & \\
\ll & \int_{2}^{X}\left(x \sum_{h \leq H} \sum_{h<m \leq D} d(m) d(m+h)(m(m+h))^{-3 / 4}\right. \\
& \left.\times(\sqrt{m+h}-\sqrt{m})^{-1} \sin \theta_{m, m+h}\right)^{r} d x+c^{r} X^{r+1} .
\end{aligned}
$$

As in the previous two cases, we multiply out the $r$-fold product in the integrand and then interchange the integration with the multiple summation. Let $\eta_{i}=\sqrt{m_{i}+h_{i}}-\sqrt{m_{i}}$. Then by (4.7) we have

$$
\begin{aligned}
\int_{X_{0}}^{X} x^{r} \prod_{i=1}^{r} \sin \left(\theta_{m_{i}, m_{i}+h_{i}}\right) d x & \\
& \ll 2^{-r} \sum_{ \pm} \min \left(X^{r+1}, X^{r+1 / 2}\left|\eta_{1} \pm \eta_{2} \pm \ldots \pm \eta_{r}\right|^{-1}\right) .
\end{aligned}
$$

Moreover,

$$
\begin{aligned}
d(m) d(m+h)(m(m+h))^{-3 / 4}(\sqrt{m+h}-\sqrt{m})^{-1} & \\
& \ll d(m) d(m+h) m^{-1} h^{-1}=g(m, h),
\end{aligned}
$$

say. We deduce from (4.13) that

$$
\begin{aligned}
\int_{2}^{X}\left(S_{6}(x)\right)^{r} d x \ll & 2^{-r} \sum_{ \pm} \sum_{h_{i} \leq X^{\tau}} \sum_{h_{i}<m_{i} \leq\left(h_{i} \sqrt{X}\right)^{7 / 4}} \prod_{i=1}^{r} g\left(m_{i}, h_{i}\right) \\
& \times \min \left(X^{r+1}, X^{r+1 / 2}\left|\eta_{1} \pm \eta_{2} \pm \ldots \pm \eta_{r}\right|^{-1}\right) \\
& +c^{r} X^{r+1} .
\end{aligned}
$$

In order to obtain (4.12), it is therefore sufficient to prove that

$$
\begin{array}{r}
\sum_{h_{i} \leq X^{\tau}} \sum_{h_{i}<m_{i} \leq\left(h_{i} \sqrt{X}\right)^{7 / 4}} \prod_{i=1}^{r} g\left(m_{i}, h_{i}\right) \min \left(X, X^{1 / 2}\left|\eta_{1} \pm \eta_{2} \pm \ldots \pm \eta_{r}\right|^{-1}\right) \\
\ll(c r)^{4 r} X
\end{array}
$$

for each possible combination of the \pm signs. Let us consider one such combination and write $\eta^{\prime}= \pm \eta_{2} \pm \ldots \pm \eta_{r}$ for short. Then the part of the sum in (4.15) for which $\left|\eta_{1}+\eta^{\prime}\right|>X^{-1 / 2+\delta}$ is obviously

$$
\ll X^{1-\delta}\left(\sum_{h \leq X^{\tau}} \sum_{h<m \leq(h \sqrt{X})^{7 / 4}} g(m, h)\right)^{r} \ll X^{1-\delta}\left(c \log ^{4} X\right)^{r},
$$

by (4.14) and Lemma 4(b). According to (4.2), this is bounded by $X(c r)^{4 r}$ 
and hence is acceptable. It remains to prove the inequality

$$
\sum_{h_{i} \leq X^{\tau}} \sum_{\substack{h_{i}<m_{i} \leq\left(h_{i} \sqrt{X}\right)^{7 / 4} \\\left|\eta_{1}+\eta^{\prime}\right| \leq X^{-1 / 2+\delta}}} \prod_{i=1}^{r} g\left(m_{i}, h_{i}\right) \ll(c r)^{4 r} .
$$

Without loss of generality, we may assume $m_{1}$ to be the largest among all the $m_{i}$ 's. Let $h_{1}, \ldots, h_{r}, m_{2}, \ldots, m_{r}$ be given and consider the sum

$$
\sum_{\substack{m_{1} \\\left|\eta_{1}+\eta^{\prime}\right| \leq X^{-1 / 2+\delta}}} g\left(m_{1}, h_{1}\right) .
$$

The number $m_{1}$ falls in the interval $\left[\max \left(h_{1}, m_{2}, \ldots, m_{r}\right),\left(h_{1} \sqrt{X}\right)^{7 / 4}\right]$ which we can cover by abutting intervals of the form $\left[z_{j}, 2 z_{j}\right]$ with

$$
\left(h_{1} m_{2} \ldots m_{r}\right)^{1 / r} \leq z_{j} \leq\left(h_{1} \sqrt{X}\right)^{7 / 4} .
$$

The condition $\left|\eta_{1}+\eta^{\prime}\right| \leq X^{-1 / 2+\delta}$ stipulates that $\sqrt{m_{1}+h_{1}}-\sqrt{m_{1}}$ lies in an interval of length $2 X^{-1 / 2+\delta}$. For fixed $h_{1}$ such that $h_{1} \ll y, \sqrt{y+h_{1}}-\sqrt{y}$ is a monotonically decreasing function of $y$ with derivative $\approx-\frac{1}{4} h_{1} y^{-3 / 2}$. Hence

$$
\#\left\{m_{1} \in\left[z_{j}, 2 z_{j}\right]:\left|\eta_{1}+\eta^{\prime}\right| \leq X^{-1 / 2+\delta}\right\} \ll h_{1}^{-1} z_{j}^{3 / 2} X^{-1 / 2+\delta}+1 .
$$

The sum in (4.17) is therefore

$$
\begin{aligned}
& \leq \sum_{j} \sum_{\substack{m_{1} \in\left[z_{j}, 2 z_{j}\right] \\
\left|\eta_{1}+\eta^{\prime}\right| \leq X^{-1 / 2+\delta}}} d\left(m_{1}\right) d\left(m_{1}+h_{1}\right) m_{1}^{-1} h_{1}^{-1} \\
& \ll \sum_{j} z_{j}^{\delta} z_{j}^{-1} h_{1}^{-1}\left(h_{1}^{-1} z_{j}^{3 / 2} X^{-1 / 2+\delta}+1\right),
\end{aligned}
$$

since for $h_{1}<m_{1}$ and $m_{1} \in\left[z_{j}, 2 z_{j}\right]$, we have $d\left(m_{1}\right) d\left(m_{1}+h_{1}\right) \ll z_{j}^{\delta}$. By (4.18) the last sum over $j$ is

$$
\begin{aligned}
& \ll X^{-1 / 2+\delta} h_{1}^{-2}\left(h_{1} \sqrt{X}\right)^{(7 / 4)(1 / 2+\delta)}+h_{1}^{-1}\left(h_{1} m_{2} \ldots m_{r}\right)^{(1 / r)(-1+\delta)} \\
& \ll X^{-\delta} h_{1}^{-1-\delta}+h_{1}^{-1-1 /(2 r)}\left(m_{2} \ldots m_{r}\right)^{-1 /(2 r)} .
\end{aligned}
$$

Summing this for $h_{1} \leq X^{\tau}$ and then inserting into (4.16), we see that the multiple sum in (4.16) is

$$
\begin{aligned}
& \ll \sum_{\substack{h_{i} \leq X^{\tau} \\
i \geq 2}} \sum_{\substack{h_{i}<m_{i} \leq\left(h_{i} \sqrt{X}\right)^{7 / 4} \\
i \geq 2}} \prod_{i=2}^{r} g\left(m_{i}, h_{i}\right)\left(X^{-\delta}+r\left(m_{2} \ldots m_{r}\right)^{-1 /(2 r)}\right) \\
& \ll X^{-\delta}\left(\sum_{h \leq X^{\tau}} \sum_{\substack{h<m \leq(h \sqrt{X})^{7 / 4} \\
h(m, h)}} g(m-1\right.
\end{aligned}
$$




$$
\begin{aligned}
& \quad+r\left(\sum_{h \leq X^{\tau}} \sum_{h<m \leq(h \sqrt{X})^{7 / 4}} m^{-1 /(2 r)} g(m, h)\right)^{r-1} \\
& \ll X^{-\delta}\left(c \log ^{4} X\right)^{r}+(c r)^{4 r},
\end{aligned}
$$

by (4.14) and Lemma 4(b). In view of (4.2), this yields the desired bound for the multiple sum in (4.16) and (4.15) thus follows.

Finally, we consider the integral $\int_{2}^{X}\left(S_{7}(x)\right)^{r} d x$. Using the same argument as for $S_{6}(x)$, we find from (3.12) that

$$
\begin{aligned}
S_{7}(x)= & \left(4 \pi^{3}\right)^{-1} x \sum_{H<h \leq M / 2} \sum_{h<m \leq M-h} d(m) d(m+h)(m(m+h))^{-3 / 4} \\
& \times(\sqrt{m+h}-\sqrt{m})^{-1} \sin \theta_{m, m+h}+\mathcal{O}(x) .
\end{aligned}
$$

In obtaining the $\mathcal{O}$-term, we have used (3.13) and $M=x^{7}$. Let $Y$ be any large number not exceeding $X / 2$. We shall prove $(4.1)_{7}$ by showing that

$$
\int_{Y}^{2 Y}\left(S_{7}(x)\right)^{r} d x \ll(c r)^{4 r} Y^{r+1} .
$$

In view of (4.19), this will follow from the inequality

$$
\begin{aligned}
\int_{Y}^{2 Y}\left(x \sum_{H<h \leq M / 2} \sum_{h<m \leq M-h} d(m) d(m+h)(m(m+h))^{-3 / 4}\right. \\
\left.\quad \times(\sqrt{m+h}-\sqrt{m})^{-1} \sin \theta_{m, m+h}\right)^{r} d x \ll(c r)^{4 r} Y^{r+1} .
\end{aligned}
$$

This integral is essentially the same as that on the right hand side of (4.13) except for the different ranges for $h$ and $m$. So following the same argument there, we reduce the proof of (4.20) to proving the following:

$$
\begin{aligned}
& \sum_{Y^{\tau}<h_{i}<\frac{1}{2}(2 Y)^{7}} \sum_{\substack{h_{i}<m_{i} \leq(2 Y)^{7}-h_{i}\\
}} \prod_{i=1}^{r} g\left(m_{i}, h_{i}\right) \\
& \times \min \left(Y, Y^{1 / 2}\left|\eta_{1} \pm \eta_{2} \pm \ldots \pm \eta_{r}\right|^{-1}\right) \ll(c r)^{4 r} Y
\end{aligned}
$$

for each combination of the \pm signs (cf. (4.14) and (4.15)). Again we fix one such combination of the \pm signs and put $\eta^{\prime}= \pm \eta_{2} \pm \ldots \pm \eta_{r}$. The part of the above sum for which $\left|\eta_{1}+\eta^{\prime}\right|>Y^{-1 / 2+\delta}$ is

$$
\begin{aligned}
& \ll Y^{1-\delta}\left(\sum_{Y^{\tau}<h<\frac{1}{2}(2 Y)^{7}} \sum_{h<m \leq(2 Y)^{7}-h} g(m, h)\right)^{r} \\
& \ll Y^{1-\delta}\left(c \log ^{4} Y\right)^{r} \leq Y(c r)^{4 r},
\end{aligned}
$$

by (4.14), Lemma 4 (b) and (4.2). 
For given $m_{1}, m_{2}, \ldots, m_{r}, h_{2}, \ldots, h_{r}$, the condition $\left|\eta_{1}+\eta^{\prime}\right| \leq Y^{-1 / 2+\delta}$ says that $\sqrt{m_{1}+h_{1}}$ lies in an interval of length $2 Y^{-1 / 2+\delta}$. As $\sqrt{m_{1}+y}$ is an increasing function of $y$ with derivative $\approx \frac{1}{2} m_{1}^{-1 / 2}$, there are $\ll Y^{-1 / 2+\delta} \sqrt{m_{1}}$ +1 values of $h_{1}$ satisfying the condition $\left|\eta_{1}+\eta^{\prime}\right| \leq 2 Y^{-1 / 2+\delta}$. Thus

$$
\begin{aligned}
\sum_{\substack{Y^{\tau}<h_{1}<\frac{1}{2}(2 Y)^{7} \\
\left|\eta_{1}+\eta^{\prime}\right| \leq 2 Y^{-1 / 2+\delta}}} & \sum_{\substack{h_{1}<m_{1} \leq(2 Y)^{7}-h_{1} \\
\ll}} g\left(m_{1}, h_{1}\right) \\
& \sum_{\substack{Y^{\tau}<m_{1} \leq(2 Y)^{7} \\
\left|\eta_{1}+\eta^{\prime}\right| \leq 2 Y^{-1 / 2+\delta}}} \sum_{Y^{\tau}<h_{1}<m_{1}}^{-1+\delta} h_{1}^{-1} \\
\ll & \sum_{Y^{\tau}<m_{1} \leq(2 Y)^{7}} m_{1}^{-1+\delta} Y^{-\tau}\left(Y^{-1 / 2+\delta} \sqrt{m_{1}}+1\right) \ll Y^{-\delta},
\end{aligned}
$$

by (3.13). Hence the part of the sum (4.21) for which $\left|\eta_{1}+\eta^{\prime}\right| \leq 2 Y^{-1 / 2+\delta}$ is

$$
\begin{aligned}
& \ll Y^{1-\delta}\left(\sum_{Y^{\tau}<h<\frac{1}{2}(2 Y)^{7}} \sum_{h<m \leq(2 Y)^{7}-h} g(m, h)\right)^{r-1} \\
& \ll Y^{1-\delta}\left(c \log ^{4} Y\right)^{r} \ll(c r)^{4 r} Y,
\end{aligned}
$$

by (4.14), Lemma 4(b) and (4.2). This and (4.22) prove (4.21) and we have thus established that

$$
\int_{2}^{X}\left(S_{7}(x)\right)^{r} d x \ll X^{1+r}(c r)^{4 r} .
$$

This completes the proof of $(4.1)_{j}$ and hence our Main Theorem is proved as well.

\section{References}

[1] J. L. Hafner, New omega theorems for two classical lattice point problems, Invent. Math. 63 (1981), 181-186.

[2] D. R. Heath-Brown, The fourth power moment of the Riemann Zeta-function, Proc. London Math. Soc. (3) 38 (1979), 385-422.

[3] A. Ivić, The Riemann Zeta-Function, Wiley-Interscience, New York, 1985.

[4] - Lectures on Mean Values of the Riemann Zeta-Function, Lectures on Math. and Physics 82, Tata Inst. Fund. Res., Springer, Bombay, 1991.

[5] A. Ivić and M. Ouellet, Some new estimates in the Dirichlet divisor problem, Acta Arith. 52 (1989), 241-253.

[6] Y. K. Lau and K. M. Tsang, Mean square of the remainder term in the Dirichlet divisor problem, J. Théorie de Nombres de Bordeaux, to appear. 
[7] Y. Motohashi, The binary additive divisor problem, Ann. Sci. École Norm. Sup. 27 (1994), 529-572.

[8] E. Preissmann, Sur la moyenne quadratique du terme de reste du problème du cercle, C. R. Acad. Sci. Paris Sér. I 306 (1988), 151-154.

[9] E. C. Titchmarsh, The Theory of the Riemann Zeta-Function, 2nd ed. revised by D. R. Heath-Brown, Oxford Univ. Press, Oxford, 1986.

[10] K. C. Tong, On divisor problems III, Acta Math. Sinica 6 (1956), 515-541.

[11] G. N. Watson, A Treatise on the Theory of Bessel Functions, 2nd ed., Cambridge University Press, 1962.

DEPARTMENT OF MATHEMATICS

UNIVERSITY OF HONG KONG

POKFULAM ROAD

HONG KONG 